\title{
Instanton effects on charmonium states
}

\author{
Ulugbek Yakhshiev, ${ }^{1, *}$ Hyun-Chul Kim, ${ }^{1,2,3, \dagger}$ and Emiko Hiyama ${ }^{2,4,5, *}$ \\ ${ }^{1}$ Department of Physics, Inha University, Incheon 22212, Republic of Korea \\ ${ }^{2}$ Advanced Science Research Center, Japan Atomic Energy Agency, \\ Shirakata, Tokai, Ibaraki 319-1195, Japan \\ ${ }^{3}$ School of Physics, Korea Institute for Advanced Study (KIAS), Seoul 02455, Republic of Korea \\ ${ }^{4}$ Department of Physics, Kyushu University, 819-0395 Fukuoka, Japan \\ ${ }^{5}$ RIKEN Nishina Center, RIKEN, 2-1 Hirosawa, 351-0115 Saitama, Japan
}

(Received 14 November 2018; published 28 December 2018)

\begin{abstract}
The instanton effects on the charmonium spectrum are discussed in the framework of the nonrelativistic potential model. The results from the constituent quark model without inclusion of instanton effects are compared with the results for the potential from the constituent quark model plus the contribution from the instanton liquid model. We consider two models with the corresponding instanton potentials and discuss their relevance to explanations of the origin of phenomenological parameters used in the nonrelativistic potential models. We also present the universal instanton potential in a parametrized form, which can be useful in practical calculations.
\end{abstract}

DOI: $10.1103 /$ PhysRevD.98.114036

\section{INTRODUCTION}

The physics of charmonia has entered a new era, since the finding of the first narrow exotic charmonium was reported by the Belle Collaboration [1]. Many narrow exotic charmonium states have been consecutively observed [2-13] and were coined collectively the XYZ mesons (see recent reviews $[14,15])$. These new exotic charmonium states have drawn considerable attention (see, e.g., the reviews in Refs. [16-21]) and have also brought about a multifaceted viewpoint of conventional charmonium states.

Theoretically, the quantum-mechanical potential models provide an easy but very effective way of describing the charmonium spectrum [17-19,22,23]. In a standard approach, there are basically two main contributions to the heavy-quark potential for the charmonium system: the Coulomb-like potential and the phenomenological quark-confining one. The Coulomb-like potential originates from one-gluon exchange (OGE) between a heavy quark $(Q)$ and a heavy antiquark $(\bar{Q})$ [24-27], based on perturbative quantum chromodynamics (pQCD). Note that the static Coulomb-like potential was scrutinized already

\footnotetext{
*yakhshiev@inha.ac.kr

hchkim@inha.ac.kr

"hiyama@phys.kyushu-u.ac.jp
}

Published by the American Physical Society under the terms of the Creative Commons Attribution 4.0 International license. Further distribution of this work must maintain attribution to the author(s) and the published article's title, journal citation, and DOI. Funded by SCOAP . to higher-order corrections from pQCD [28-32]. By the nature of $\mathrm{pQCD}$, the Coulomb-like interactions are supposed to govern the short-range physics of the charmonia. At large distances the strength of the Coulomb-like interaction decreases. However, the presence of the quark-confining potential makes the strength of the total interaction increase. This is due to the fact that any quark inside a charmonium is ordained to be confined in it, so effects of the quark confinement [33] are necessarily involved. The heavy-quark potential for the quark confinement can be obtained at least phenomenologically from the Wilson loop, which rises linearly at large distances [22,23]. On the other hand, as the quark and the antiquark start to recede from each other, certain nonperturbative contributions come into play.

Recently, we examined other nonperturbative effects on the mass spectrum of the charmonia from the instanton vacuum of QCD [34]. The central part of the heavy-quark potential was already derived by Diakonov et al. [35], based on the instanton liquid model for the QCD vacuum [36-38]. The spin-dependent part of the instanton-induced potential can be easily obtained by employing the method of Eichten and Feinberg [39]. There are two intrinsic parameters of characterizing the instanton vacuum, i.e., the average size of the instanton $\rho$ and the average distance $R$ between instantons. Their numerical values were estimated to be $\rho \approx 0.33 \mathrm{fm}$ and $R \approx 1 \mathrm{fm}[36,37,40]$. However, these values are just the approximated ones. For example, the authors of Refs. [41-43] considered $1 / N_{c}$ meson-loop contributions in the light-quark sector and found it necessary to readjust the values of parameters 
as $\rho \simeq 0.35 \mathrm{fm}$ and $R \simeq 0.86 \mathrm{fm}$. In Ref. [34], the authors scrutinized the dependence of the heavy-quark potential from the instanton vacuum. In the present work, we combine the instanton-induced heavy-quark potential with the Coulomb-like and quark-confinement potentials and investigate explicitly the instanton effects on the mass spectrum of the charmonia.

The paper is organized as follows. In Sec. II, we explain briefly the nonrelativistic heavy-quark potential model consisting of the color Coulomb-like potential and the linear scalar potential for quark confinement [44] and discuss the contribution to the total heavy-quark potential from the instanton vacuum. In Sec. III, the Gaussian expansion method for a numerical calculation will be briefly described. In Sec. IV we present the results and discuss them. The final Sec. V is devoted to the summary and conclusions of this work.

\section{HEAVY-QUARK POTENTIAL}

The standard heavy-quark potential consists of four different terms, written as

$$
\begin{aligned}
V_{Q \bar{Q}}(\boldsymbol{r})= & V_{C}(r)+V_{S S}(r)\left(\boldsymbol{S}_{Q} \cdot \boldsymbol{S}_{\bar{Q}}\right) \\
& +V_{L S}(r)(\boldsymbol{L} \cdot \boldsymbol{S}) \\
& +V_{T}(r)\left[3\left(\boldsymbol{S}_{Q} \cdot \boldsymbol{n}\right)\left(\boldsymbol{S}_{\bar{Q}} \cdot \boldsymbol{n}\right)-\boldsymbol{S}_{Q} \cdot \boldsymbol{S}_{\bar{Q}}\right],
\end{aligned}
$$

where $V_{C}, V_{S S}, V_{L S}$, and $V_{T}$ represent, respectively, the central, spin-spin, spin-orbit, and tensor parts of the heavyquark potential. $\boldsymbol{S}_{Q}, \boldsymbol{S}_{\bar{Q}}$, and $\boldsymbol{L}$ denote the spin operator of a heavy quark, that of a heavy antiquark, and the relative orbital angular momentum operator, respectively. The unit vector $\boldsymbol{n}$ in three-dimensional ordinary space is chosen in the direction of the line joining the centers of the heavy quark and heavy antiquark. All contributions to the total $Q \bar{Q}$ potential can be constructed within the framework of nonrelativistic potential approaches. As mentioned previously, the color Coulomb-like vector potential arises from OGE between $Q$ and $\bar{Q}$, while the linear scalar potential is constructed phenomenologically from the area law of the Wilson loop [33] for the quark confinement. We denote the corresponding OGE vector + scalar confining potential as $V^{(\mathrm{P})}$. In addition, we introduce the nonperturbative potential $V^{(\mathrm{NP})}$ derived from the instanton vacuum [34]. Consequently, the heavy-quark potential in the present work is written as

$$
V(r)=V^{(\mathrm{P})}(r)+V^{(\mathrm{NP})}(r) .
$$

We will compute the mass spectrum of the charmonia based on the potential in Eq. (2). However, we want to emphasize that we will not carry out a fine-tuning to reproduce the experimental data, since it is of greater importance to understand the nonperturbative effects coming from the instanton-induced potential and physical implications of the parameters involved.

\section{A. Heavy-quark potential}

A nonrelativistic potential model [44] provides a minimal theoretical framework to describe the charmonia. The central part of the heavy-quark potential is expressed as

$$
V_{C}^{(\mathrm{P})}(r)=\kappa r-\frac{4 \alpha_{s}}{3 r},
$$

where the first term expresses the linear scalar potential for the quark confinement and the second one comes from OGE, respectively. Here $\kappa$ stands for the parameter of the string tension, of which the numerical value can be approximately determined by reproducing the mass spectrum of the charmonia. The parameter $\alpha_{s}$ denotes the running strong coupling constant of $\mathrm{pQCD}$, of which the value is theoretically well known. We fix the scale of $\alpha_{s}$ at the mass of the charm quark. We will discuss physical implications of these two parameters later.

The spin-dependent parts can be obtained from the central potential (3)

$$
\begin{gathered}
V_{S S}^{(\mathrm{P})}(r)=\frac{32 \pi \alpha_{s}}{9 m_{Q}^{2}} \tilde{\delta}_{\sigma}(r), \\
V_{L S}^{(\mathrm{P})}(r)=\frac{1}{2 m_{Q}^{2}}\left(\frac{4 \alpha_{s}}{r^{3}}-\frac{\kappa}{r}\right), \\
V_{T}^{(\mathrm{P})}(r)=\frac{4 \alpha_{s}}{m_{Q}^{2} r^{3}},
\end{gathered}
$$

which appear from the next-to-leading order in the expansion of the heavy quark mass $m_{Q}$. So, the spin-dependent potentials are proportional to $1 / m_{Q}^{2}$, respectively. In the present work, the value of the charm-quark mass $m_{c}$ will be determined by including the instanton effects. A smeared Dirac delta function $\tilde{\delta}_{\sigma}$ in Eq. (4) is written in the Gaussian form

$$
\tilde{\delta}_{\sigma}(r)=\left(\frac{\sigma}{\sqrt{\pi}}\right)^{3} e^{-\sigma^{2} r^{2}}
$$

where $\sigma$ is the smearing parameter that can be determined phenomenologically.

\section{B. Instanton-induced potential}

In addition to the potential given in Eq. (3), we introduce the instanton-induced potential, which was already derived in Refs. [34,35]. The explicit form of the potential is expressed as 


$$
V_{C}^{(\mathrm{NP})}(r)=\frac{4 \pi \rho^{3}}{N_{c} R^{4}} \mathcal{I}\left(\frac{r}{\rho}\right)
$$

where $N_{c}$ denotes the number of colors, and $\rho$ and $R$ stand for the average instanton size and average interinstanton distance, respectively. The dimensionless integral $\mathcal{I}(x)$ is given as a function of the dimensionless variable $x$ :

$$
\begin{aligned}
\mathcal{I}(x)= & \int_{0}^{\infty} y^{2} d y \int_{-1}^{1} d t\left\{1-\cos \left(\frac{\pi y}{\sqrt{y^{2}+1}}\right)\right. \\
& \times \cos \left(\pi \sqrt{\frac{y^{2}+x^{2}+2 x y t}{y^{2}+x^{2}+2 x y t+1}}\right) \\
& -\frac{y+x t}{\sqrt{y^{2}+x^{2}+2 x y t}} \sin \left(\frac{\pi y}{\sqrt{y^{2}+1}}\right) \\
& \left.\times \sin \left(\pi \sqrt{\frac{y^{2}+x^{2}+2 x y t}{y^{2}+x^{2}+2 x y t+1}}\right)\right\} .
\end{aligned}
$$

Though it is possible to compute Eq. (9) numerically given $x$, it is more convenient to parametrize the integral $\mathcal{I}(x)$ such that one can easily make it useful for a practical calculation. We obtain a suitable parametrization as follows:

$\tilde{\mathcal{I}}(x)=\mathcal{I}_{0}\left[1+\sum_{i=1}^{3} a_{i} x^{2(i-1)} e^{-b_{i} x^{2}}+\frac{a_{4}}{x}\left(1-e^{-b_{4} x^{3}}\right)\right]$,

where the prefactor $\mathcal{I}_{0}$ is expressed in terms of the Bessel functions

$$
\mathcal{I}_{0}=-\frac{2 \pi^{3}}{3}\left(J_{0}(\pi)+\frac{1}{\pi} J_{1}(\pi)\right)
$$

and the parameters $a_{i}$ and $b_{i}$ are summarized in the matrix forms

$$
a=\left(\begin{array}{l}
-1 \\
0.10184 \\
0.00064 \\
-1.11267
\end{array}\right), \quad b=\left(\begin{array}{l}
0.25135 \\
0.70255 \\
0.18625 \\
0.04644
\end{array}\right) \text {. }
$$

One can inspect whether the parametrized function $\tilde{\mathcal{I}}$ in Eq. (10) yields the correct limiting values of the original integral $\mathcal{I}(x)$ in Eq. (9) at $x=0$ and $x \rightarrow \infty$. That is, the relations

$$
\begin{aligned}
\lim _{x \rightarrow 0} \mathcal{I}(x) & =\lim _{x \rightarrow 0} \tilde{\mathcal{I}}(x)=0, \\
\lim _{x \rightarrow \infty} \mathcal{I}(x) & =\lim _{x \rightarrow \infty} \tilde{\mathcal{I}}(x)=\mathcal{I}_{0}
\end{aligned}
$$

are well satisfied. At small $x \ll 1, \mathcal{I}(x)$ can be analytically evaluated as

$$
\begin{aligned}
\mathcal{I}(x) \simeq & \frac{\pi^{2}}{3}\left[\frac{\pi}{16}-J_{1}(2 \pi)\right] x^{2} \\
& -\pi\left[\frac{\pi^{2}\left(438+7 \pi^{2}\right)}{30720}+\frac{J_{2}(2 \pi)}{80}\right] x^{4} \\
= & 1.34467 x^{2}-0.500508 x^{4},
\end{aligned}
$$

while the parametrization of Eq. (10) gives the result

$$
\begin{aligned}
\tilde{\mathcal{I}}(x) \simeq & \mathcal{I}_{0}\left(-a_{1} b_{1}+a_{2}+a_{4} b_{4}\right) x^{2} \\
& +\frac{\mathcal{I}_{0}}{2}\left(a_{1} b_{1}^{2}-2 a_{2} b_{2}+2 a_{3}\right) x^{4} \\
= & 1.3316 x^{2}-0.452657 x^{4} .
\end{aligned}
$$

At large $x$, the limiting results of $\mathcal{I}(x)$ and $\tilde{\mathcal{I}}(x)$ are produced as

$$
\begin{gathered}
\mathcal{I}(x) \simeq \mathcal{I}_{0}-\frac{\pi^{2}}{2 x}=4.41625-\frac{4.9348}{x}, \\
\tilde{\mathcal{I}}(x) \simeq \mathcal{I}_{0}\left(1+\frac{a_{4}}{x}\right)=4.41625-\frac{4.91384}{x},
\end{gathered}
$$

respectively. From Eqs. (13)-(16), one can see that the corresponding coefficients of the asymptotic forms are very close to each other.

In general, the parametrization in Eq. (10) interpolates the numerical value of the integral $\mathcal{I}(x)$ with a very good accuracy in the whole range of $x$. In Fig. 1, the numerical result of Eq. (9) is compared with that from the parametrization in Eq. (10). As shown in Fig. 1, they overlap completely in the whole range of $x$. The maximal value of a relative error is located only in the small $x$ region and does not exceed the value

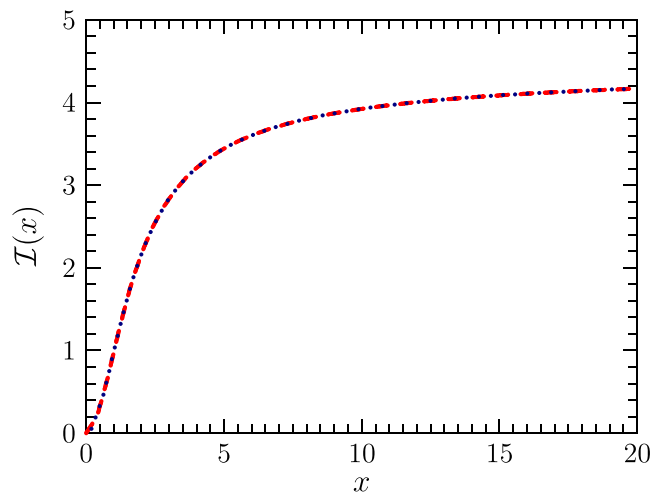

FIG. 1. The dimensionless integral $\mathcal{I}(x)$ from the instanton vacuum. The numerical result of Eq. (9) is depicted as the red dashed curve, whereas that of the parametrization given in Eq. (10) is drawn as the blue dotted curve. 


$$
\begin{aligned}
& \lim _{x \rightarrow 0} \frac{\mathcal{I}(x)-\tilde{\mathcal{I}}(x)}{\mathcal{I}(x)}=1 \\
& \quad-\mathcal{I}_{0}\left(b_{1}+a_{2}+a_{4} b_{4}\right)\left\{\frac{\pi^{2}}{3}\left[\frac{\pi}{16}-J_{1}(2 \pi)\right]\right\}^{-1} \\
& =0.00972 .
\end{aligned}
$$

At large values of $x \rightarrow \infty$, the relative error decreases according to the formula

$$
\frac{\mathcal{I}(x)-\tilde{\mathcal{I}}(x)}{\mathcal{I}(x)} \approx-\frac{0.00475}{x-1.11742}
$$

The spin-dependent parts of the heavy-quark potential from the instanton vacuum can be obtained from the central part according to the following formulas:

$$
\begin{gathered}
V_{S S}^{(\mathrm{NP})}(r)=\frac{1}{3 m_{Q}^{2}} \nabla^{2} V_{C}^{(\mathrm{NP})}(r), \\
V_{L S}^{(\mathrm{NP})}(r)=\frac{1}{2 m_{Q}^{2}} \frac{1}{r} \frac{d V_{C}^{(\mathrm{NP})}(r)}{d r}, \\
V_{T}^{(\mathrm{NP})}(r)=\frac{1}{3 m_{Q}^{2}}\left(\frac{1}{r} \frac{d V_{C}^{(\mathrm{NP})}(r)}{d r}-\frac{d^{2} V_{C}^{(\mathrm{NP})}(r)}{d r^{2}}\right) .
\end{gathered}
$$

Using the parametrization that we introduced above, we can compute almost all integrations for the instantoninduced potential analytically. The merit of the parametrization given in Eq. (10) is not limited to the simple calculation of the integration $\mathcal{I}(x)$. In fact, the instantoninduced central potential is expressed in terms of $\mathcal{I}(x)$ with all other instanton parameters factored out. It means that $\mathcal{I}(x)$ can be used in the universal way for any set of the instanton parameters. So, the spin-dependent potentials in Eqs. (19)-(21) can also be expressed in terms of $\mathcal{I}(x)$ for any heavy-quark degrees of freedom, i.e., for the charmonia or bottomonia.

The instanton liquid model for the QCD vacuum has two important intrinsic parameters: the average size of the instanton $\rho$ and the average interdistance $R$ between instantons. In fact, the $Q \bar{Q}$ potential is sensitive to them. Thus, we will consider three different sets of instanton parameters by changing them in a permissible manner. Model I (M-I) uses the original values of the instanton parameters: $\rho \simeq 0.33 \mathrm{fm}$ and $R \simeq 1 \mathrm{fm}$. However, $\rho$ and $R$ can be changed in a different situation. For example, the authors of Refs. [41-43] considered the $1 / N_{c}$ meson-loop contributions in the light-quark sector and found it necessary to readjust them as $\rho \simeq 0.35 \mathrm{fm}$ and $R \simeq 0.856 \mathrm{fm}$. Model IIa (M-IIa) employs these values as in Ref. [34]. In lattice $\mathrm{QCD}$, the instanton vacuum was simulated and the following values were suggested: $\rho \approx 0.36 \mathrm{fm}$ and
$R \approx 0.89 \mathrm{fm}$ [45-48], which are almost the same as those with the $1 / N_{c}$ meson-loop corrections. Therefore, the model with this set is referred to as model IIb (M-IIb), also as in Ref. [34]. The parameter dependence of the potential can be easily understood from the form of the leading-order potential expressed in Eq. (8). While the prefactor $\rho^{3} / R^{4} N_{c}$, which includes both the parameters, governs the overall strength of the potential, its range is dictated only by the average instanton size $\rho$ through the dimensionless integral $\mathcal{I}(r / \rho)$.

\section{NUMERICAL METHOD}

In order to evaluate the bound states in the spectrum of the quarkonia, we have to solve the Schrödinger equation [49]

$$
(\hat{H}-E)\left|\Psi_{J_{3}}\right\rangle=0,
$$

where $\hat{H}$ is the Hamiltonian operator and $\left|\Psi_{J_{3}}\right\rangle$ represents the corresponding state vector with the total angular momentum $J$ and its third component $J_{3}$. The projection of the state vector $\left\langle\boldsymbol{r} \mid \Psi_{J_{3}}\right\rangle$ will reproduce the representation of the Hamiltonian in coordinate space,

$$
\hat{H}(\boldsymbol{r})=-\frac{\hbar^{2}}{m_{Q}} \nabla^{2}+V_{Q \bar{Q}}(\boldsymbol{r}),
$$

where $\mu_{Q}$ arises from the reduced mass of the quarkonium system. The matrix elements of the $Q \bar{Q}$ potential in the standard basis $\left|{ }^{2 S+1} L_{J}\right\rangle$, which is given in terms of the total spin $S$, the orbital angular momentum $L$, and the total angular momentum $J$ satisfying the relation $\boldsymbol{J}=\boldsymbol{L}+\boldsymbol{S}$, are obtained as

$$
\begin{aligned}
V_{Q \bar{Q}}(r)= & \left\langle{ }^{2 S+1} L_{J}\left|V_{Q \bar{Q}}(\boldsymbol{r})\right|{ }^{2 S+1} L_{J}\right\rangle \\
= & V(r)+\left[\frac{1}{2} S(S+1)-\frac{3}{4}\right] V_{S S}(r) \\
& +\langle\boldsymbol{L} \cdot \boldsymbol{S}\rangle V_{L S}(r)+\left\langle\boldsymbol{\Omega}_{T}\right\rangle V_{T}(r) .
\end{aligned}
$$

Here the matrix element of the tensor operator is obtained to be

$$
\begin{aligned}
\left\langle\boldsymbol{\Omega}_{T}\right\rangle= & \frac{S(S+1) L(L+1)}{3(2 L-1)(2 L+3)} \\
& -\frac{2\langle\boldsymbol{L} \cdot \boldsymbol{S}\rangle[2\langle\boldsymbol{L} \cdot \boldsymbol{S}\rangle+1]}{4(2 L-1)(2 L+3)}
\end{aligned}
$$

and $\langle\boldsymbol{L} \cdot \boldsymbol{S}\rangle$ is given by the expression

$$
\langle\boldsymbol{L} \cdot \boldsymbol{S}\rangle=\frac{1}{2}[J(J+1)-L(L+1)-S(S+1)] .
$$

The corresponding radial part of the wave function for a given orbital angular momentum $L$ is a solution of the Schrödinger equation 


$$
\left(-\frac{\hbar^{2}}{m_{Q}} \nabla^{2}+V_{Q \bar{Q}}(r)-E\right) \psi_{L L_{3}}(\boldsymbol{r})=0
$$

where an angular part of the wave function $\psi_{L L_{3}}(\boldsymbol{r})$ is represented in terms of the spherical harmonics $Y_{L L_{3}}(\hat{\boldsymbol{r}})$.

In order to solve Eq. (27) numerically, we will follow the Gaussian expansion method (see review [50]). Firstly, we expand the state vector $\left|\psi_{L L_{3}}\right\rangle$ in terms of a set of basis vectors $\left\{\left|\phi_{n L L_{3}}\right\rangle ; n=1,2, \ldots, n_{\max }\right\}$ as

$$
\left|\psi_{L L_{3}}\right\rangle=\sum_{n=1}^{n_{\max }} C_{n}^{(L)}\left|\phi_{n L L_{3}}\right\rangle
$$

Secondly, we express the basis wave functions in the spherical coordinates

$$
\phi_{n L L_{3}}(\boldsymbol{r})=\phi_{n L}^{G}(r) Y_{L L_{3}}(\hat{\boldsymbol{r}}) .
$$

The radial part of the wave function is expressed in terms of the Gaussian functions

$$
\phi_{n L}^{G}(r)=\left(\frac{2^{2 L+7 / 2} r_{n}^{-2 L-3}}{\sqrt{\pi}(2 L+1) ! !}\right)^{1 / 2} r^{L} e^{-\left(r / r_{n}\right)^{2}}
$$

where $r_{n}$ are variational parameters. One should note that the set of the wave functions $\left\{\phi_{n L L_{3}}(\boldsymbol{r}) ; n=1,2, \ldots, n_{\max }\right\}$ is properly normalized while they do not need to satisfy the orthogonality condition; i.e., they consist of a nonorthogonal basis. To obtain high accuracy by using the expansion in Eq. (28), one should optimize the set of variational parameters $\left\{r_{n} ; n=1,2, \ldots, n_{\max }\right\}$. We follow an optimization discussed in Ref. [50] and express the variational parameters by using a geometric progression:

$$
r_{n}=r_{1} a^{n-1}, \quad n=1,2, \ldots, n_{\max } .
$$

Thus, the number of variational parameters is reduced down to three, i.e., $\left\{r_{1}, a, n_{\max }\right\}$ or $\left\{r_{1}, r_{\max }, n_{\max }\right\}$.

The expansion coefficients $C_{n}^{(L)}$ in Eq. (28) and the eigenenergy $E$ are determined by employing the RayleighRitz variational principle. This leads to a generalized matrix eigenvalue problem

$$
\begin{aligned}
& \sum_{n=1}^{n_{\max }}\left(K_{m n}^{(L)}+V_{m n}^{(L)}-E N_{m n}^{(L)}\right) C_{n}^{(L)}=0, \\
& \quad m=1,2, \ldots, n_{\max },
\end{aligned}
$$

where the matrix elements of the corresponding kinetic and potential energies are obtained by

$$
\begin{gathered}
T_{m n}^{(L)}=\left\langle\phi_{m L L_{3}}\left|\frac{\hat{p}^{2}}{m_{Q}}\right| \phi_{n L L_{3}}\right\rangle=\frac{\hbar^{2}(2 L+1)}{m_{Q}\left(r_{m}^{2}+r_{n}^{2}\right)} N_{m n}^{(L)}, \\
V_{m n}^{(L)}=\left\langle\phi_{m L L_{3}}\left|V_{Q \bar{Q}}(r)\right| \phi_{n L L_{3}}\right\rangle=\frac{2^{2 L+7 / 2}}{\sqrt{\pi}(2 L+1) ! !\left(r_{m} r_{n}\right)^{L+3 / 2}} \int_{0}^{\infty} r^{2(L+1)} \exp \left\{-\frac{r^{2}\left(r_{m}^{2}+r_{n}^{2}\right)}{r_{m}^{2} r_{n}^{2}}\right\} V_{Q \bar{Q}}(r) \mathrm{d} r, \\
N_{m n}^{(L)}=\left\langle\phi_{m L L_{3}} \mid \phi_{n L L_{3}}\right\rangle=\left(\frac{2 r_{m} r_{n}}{r_{m}^{2}+r_{n}^{2}}\right)^{L+3 / 2} .
\end{gathered}
$$

Because of Eq. (31), the overlap matrix element between the nearest neighborhoods

$$
N_{n+1, n}^{(L)}=\left(\frac{2}{1+a^{2}}\right)^{L+3 / 2}
$$

is a constant that is independent of $n$. This is one of the reasons why the expansion works very well. In a practical calculation $a>1$ and for the farthest neighborhoods $(|n-m|=k \gg 1)$ an orthogonality is approximately satisfied; i.e., the corresponding overlap matrix element becomes small, $N_{m n}^{(L)} \sim 2 a^{-k-2}$.

\section{RESULTS AND DISCUSSIONS}

In the present work, we set up three different models, depending on how to fix the numerical values of the relevant parameters. The first model is merely a nonrelativistic potential model based only on the Coulomblike potential and the linear scalar one [44] without any contributions from the instanton vacuum. We will call it the model without instantons (MWOI) and list the corresponding set of parameters in Table I. We do not intend to carry out any fine-tuning to the experimental data but we concentrate on nonperturbative physics as to how the instanton-induced potentials have an effect on the charmonium spectrum. We also discuss physical implications of the parameters involved in the present model, including both the instanton parameters and other ones such as $m_{Q}, \kappa$, and $\alpha_{s}$. For example, while the charm-quark mass $m_{c}$ is often treated as a free parameter, we will not consider it as a free parameter. Once the instanton effects are taken into account, $m_{c}$ appears as a sum of the current quark mass 
TABLE I. Parameters corresponding to each model. MWOI represents the model without any instanton contributions, whereas M-I and M-IIb contain them as explained in Ref. [34].

\begin{tabular}{lcccccc}
\hline \hline The model & $\rho(\mathrm{fm})$ & $R(\mathrm{fm})$ & $\Delta m_{\mathrm{I}}(\mathrm{GeV})$ & $\alpha_{s}(\mathrm{GeV})$ & $\kappa\left(\mathrm{GeV}^{2}\right)$ & $\sigma(\mathrm{GeV})$ \\
\hline MWOI & Not applicable & Not applicable & Not applicable & 0.2068 & 0.1746 & 5.0248 \\
M-I & 0.33 & 1.00 & 0.0676 & 0.3447 & 0.1520 & 0.9331 \\
M-IIb & 0.36 & 0.89 & 0.1357 & 0.4588 & 0.1279 & 0.5650 \\
\hline \hline
\end{tabular}

$m_{c}^{\text {current }}=1.275 \mathrm{GeV}$ and the dynamical contribution to the mass arising from the instanton vacuum, $\Delta m_{\mathrm{I}}$, i.e., $m_{Q}=m_{c}^{\text {current }}+\Delta m_{\mathrm{I}}$ (see detailed discussions in Refs. [34,35,51]). The remaining three parameters $\alpha_{s}, \kappa$, and $\sigma$ will be fitted to experimentally known masses of six charmonia that are taken from the $S$-wave ones. By doing that, we fix the parameters appearing in the central part of the potentials, while those of the spin-dependent parts are automatically determined. Note again that the charm-quark mass is not a free parameter. So, the spin-orbit and tensor potentials must come out naturally as in Eqs. (5) and (20). We list the numerical values of all the parameters corresponding to each model in Table I, which were fixed as explained above.

In Table II, we present the results of each model in comparison with the experimental data [52] listed in the last column. The masses of the six $S$-wave charmonia are used as input, as noted in the second column. As shown from the results of the MWOI listed in the third column of Table II, the results on the masses of the $S$-wave charmonia are somewhat deviated from the experimental data. If one had released the charm-quark mass to be a mere free parameter, we would have described the experimental data very well. For example, various potential models adopt larger values of $m_{c}$ than in the present work, since they yield better results, compared to the experimental data. However, such a fitting procedure would obscure the physical meaning of the quark mass $m_{Q}$. We want to emphasize that the heavyquark mass itself is a physical and dynamical quantity that can also be influenced by both perturbative and nonperturbative interactions. Thus, one needs to analyze carefully the effects of the charm-quark mass on a relevant physical system. In fact, the quarkonia provides a system appropriate for scrutinizing the physical implications of the charm-quark mass. In particular, the effects from the instanton vacuum put us on the right track. Using $m_{c}$ with the instanton contribution added, we are able to fit better the three parameters to the masses of the six $S$-wave charmonia.

In the instanton liquid model of the QCD vacuum, one can estimate the instanton contribution to the current quark mass. In the light-quark systems, the generation of the mass is fully dynamical, so that almost all the constituent quark mass originates from the spontaneous breaking of chiral symmetry. When it comes to the heavy-quark systems, one also needs to consider them, though the nonperturbative effects are not as significant as in the case of the light hadrons. In Table I, the explicit values of $\Delta m_{\mathrm{I}}$ due to the instanton effects are presented for each model. The strength of the instanton effects depends on the instanton density $n \sim(\rho / R)^{4}$ and the acquired mass $\Delta m_{\mathrm{I}}$ is proportional to it (compare the value in $\mathrm{M}-\mathrm{I}$ with that in $\mathrm{M}-\mathrm{IIb}$, listed in Table I).

TABLE II. Results on the masses of the $c \bar{c}$ states, given in units of $\mathrm{MeV}$. The second column denotes explicitly those of the $S$-wave charmonia used as input to fix the parameters $\left(\alpha_{s}, \kappa\right.$, and $\sigma$ ) for each model. The third column lists the results from the original potential without the instanton-induced potentials, whereas the fourth and fifth columns show those from model I and model IIb, respectively.

\begin{tabular}{|c|c|c|c|c|c|}
\hline State & Input & MWOI & M-I & M-IIb & Exp. [52] \\
\hline$J / \psi\left(1^{3} \mathrm{~S}_{1}\right)$ & 3097 & 3084 & 3094 & 3096 & $3096.900 \pm 0.006$ \\
\hline$\eta_{c}\left(1^{1} \mathrm{~S}_{0}\right)$ & 2983 & 3027 & 2998 & 2983 & $2983.9 \pm 0.5$ \\
\hline$\psi\left(2^{3} \mathrm{~S}_{1}\right)$ & 3686 & 3635 & 3656 & 3675 & $3686.097 \pm 0.025$ \\
\hline$\eta_{c}\left(2^{1} \mathrm{~S}_{0}\right)$ & 3640 & 3590 & 3615 & 3638 & $3637.6 \pm 1.2$ \\
\hline$\psi\left(3^{3} \mathrm{~S}_{1}\right)$ & 4040 & 4067 & 4069 & 4071 & $4039 \pm 1$ \\
\hline$\eta_{c}\left(3^{1} \mathrm{~S}_{0}\right)$ & & 4026 & 4041 & 4047 & \\
\hline$\psi\left(4^{3} \mathrm{~S}_{1}\right)$ & 4415 & 4443 & 4422 & 4398 & $4421 \pm 4$ \\
\hline$\eta_{c}\left(4^{1} \mathrm{~S}_{0}\right)$ & & 4405 & 4400 & 4379 & \\
\hline$\chi_{c 2}\left(1^{3} \mathrm{P}_{2}\right)$ & & 3428 & 3607 & 3740 & $3556.17 \pm 0.07$ \\
\hline$\chi_{c 1}\left(1^{3} \mathrm{P}_{1}\right)$ & & 3437 & 3589 & 3715 & $3510.67 \pm 0.05$ \\
\hline$\chi_{c 0}\left(1^{3} \mathrm{P}_{0}\right)$ & & 3415 & 3551 & 3673 & $3414.71 \pm 0.30$ \\
\hline$h_{c}\left(1^{1} \mathrm{P}_{1}\right)$ & & 3430 & 3599 & 3727 & $3525.38 \pm 0.11$ \\
\hline$\chi_{c 2}\left(2^{3} \mathrm{P}_{2}\right)$ & & 3888 & 4039 & 4138 & $3927.2 \pm 2.6$ \\
\hline$\chi_{c 1}\left(2^{3} \mathrm{P}_{1}\right)$ & & 3890 & 4030 & 4125 & \\
\hline$\chi_{c 0}\left(2^{3} \mathrm{P}_{0}\right)$ & & 3866 & 4006 & 4098 & $3862_{-32-13}^{+26+40}$ \\
\hline$h_{c}\left(2^{1} \mathrm{P}_{1}\right)$ & & 3887 & 4039 & 4134 & \\
\hline$\chi_{c 2}\left(3^{3} \mathrm{P}_{2}\right)$ & & 4281 & 4414 & 4466 & \\
\hline$\chi_{c 1}\left(3^{3} \mathrm{P}_{1}\right)$ & & 4280 & 4394 & 4455 & \\
\hline$\chi_{c 0}\left(3^{3} \mathrm{P}_{0}\right)$ & & 4256 & 4375 & 4436 & \\
\hline$h_{c}\left(3^{1} \mathrm{P}_{1}\right)$ & & 4278 & 4402 & 4463 & \\
\hline$\psi_{3}\left(1^{3} \mathrm{D}_{3}\right)$ & & 3692 & 3830 & 3929 & \\
\hline$\psi_{2}\left(1^{3} \mathrm{D}_{2}\right)$ & & 3718 & 3836 & 3927 & $3822.2 \pm 1.2$ \\
\hline$\psi\left(1^{3} \mathrm{D}_{1}\right)$ & & 3730 & 3830 & 3914 & $3778.1 \pm 1.2$ \\
\hline$\eta_{c 2}\left(1^{1} \mathrm{D}_{2}\right)$ & & 3708 & 3837 & 3930 & \\
\hline$\psi_{3}\left(2^{3} \mathrm{D}_{3}\right)$ & & 4104 & 4238 & 4311 & \\
\hline$\psi_{2}\left(2^{3} \mathrm{D}_{2}\right)$ & & 4124 & 4242 & 4310 & \\
\hline$\psi\left(2^{3} \mathrm{D}_{1}\right)$ & & 4131 & 4241 & 4303 & $4191 \pm 5$ \\
\hline$\eta_{c 2}\left(2^{1} \mathrm{D}_{2}\right)$ & & 4116 & 4245 & 4314 & \\
\hline
\end{tabular}


While the present work mainly aims at the effects of the instanton-induced potentials, we will briefly mention which model can be most favored phenomenologically. Having simply considered the sum of the quadratic deviations of the results from the experimental data, we find that $\mathrm{M}-\mathrm{IIb}$ provides the best-fitted values for the $S$-wave charmonia. In particular, one can see that the experimental values of the four lowest-lying $S$ states are almost perfectly reproduced within M-IIb. On the other hand, as far as the whole spectrum with 15 experimentally known states given in the last column of Table II is concerned, MWOI and M-I yield better results in comparison with $\mathrm{M}$-IIb. In general, the results from the MWOI and M-I are qualitatively more or less similar to each other. However, as mentioned already, the present purpose is not to perform a fine-tuning to the experimental data but to analyze carefully the instanton effects on the charmonium spectra. Therefore, let us concentrate on the physical implications of the three potential models given in Table I.

The results of calculations show that M-I describes the mass spectrum of the charmonia quite well even up to the $D$-wave states. In particular, the mass of the $D$-wave charmonium $\psi_{2}\left(1^{3} D_{2}\right)$ is much improved by the instanton effects in M-I. On the other hand, although it gives a better fit to $S$-wave states, M-IIb does not show any improvement in comparison with the MWOI as related to $\psi_{2}\left(1^{3} D_{2}\right)$. It indicates that the original values of the instanton parameters $\rho$ and $R$ yield the better results, which are in fact expected. Those values employed in M-IIb were determined when the $1 / N_{c}$ meson-loop corrections are involved, which is not the case of the present work.

Another important issue lies in the running strong coupling constant $\alpha_{s}$. Since the Coulomb-like potential arises from OGE in pQCD, the value of $\alpha_{s}(\mu)$ should be taken from $\mathrm{pQCD}$ at a proper scale related to the charmonia. In fact, the static Coulomb potential was extensively studied within the framework of pQCD, even with higherorder corrections being taken into account [28-32]. However, a fitted value of $\alpha_{s}$ was used in many models because it is simpler and phenomenologically more favorable than that from pQCD. At the one-loop level, it is given by the expression

$$
\alpha_{s}(\mu)=\frac{4 \pi}{\beta_{0}} \frac{1}{\ln \left(\mu^{2} / \Lambda_{\mathrm{QCD}}^{2}\right)},
$$

where the $\beta$ function at the one-loop level is $\beta_{0}=\left(11 N_{c}-2 N_{f}\right) / 3$. The dimensionful parameter of $\mathrm{QCD}$ is given as $\Lambda_{\mathrm{QCD}}=0.217 \mathrm{GeV}$ [52] and $\mu$ stands for the specific scale at which the value of $\alpha_{s}$ is evaluated. It is usual to take $\mu \approx m_{c}$ for the charmonia.

The value of $\alpha_{s}$ at the one-loop level can be easily computed by using Eq. (37), once a proper scale $\mu$ is given. Considering the fact that MWOI, M-I, and M-IIb have different charm-quark masses, we can calculate the value of $\alpha_{s}$ corresponding to a specific model by using the charmquark mass of the model as its intrinsic scale. The results for M-I and M-IIb are obtained, respectively, as $\alpha_{s}(\mu=$ $1.343 \mathrm{GeV})=0.4137$ and $\alpha_{s}(\mu=1.411 \mathrm{GeV})=0.4029$, which are slightly larger or smaller than the corresponding fitted values listed in Table I, respectively. On the other hand, the value of $\alpha_{s}$ for MWOI turns out to be $\alpha_{s}(\mu=1.275 \mathrm{GeV})=0.4258$, which is approximately two times larger than the fitted value 0.2068. This comparison already demonstrates that in the presence of the instanton-induced interaction, one is allowed to use a more physical strong coupling constant for the Coulomb-like potential, based firmly on perturbative QCD.

The origin of the confining scalar potential is not much known theoretically and the string tension $\kappa$ is considered as a phenomenological parameter, though it can be related to Regge trajectories within some models [53]. The parameter $\kappa$ was often determined by the charmonium spectrum together with the effective strong coupling constant $[23,49,53]$ and the numerical value of $\kappa$ is known approximately to be $\kappa \approx 0.18 \mathrm{GeV}^{2}$ (see a review [53]). In the present work, we fix it to be $0.175 \mathrm{GeV}^{2}$ for the MWOI, which is very close to the above-given value. However, once we introduce the instanton-induced potential, we have to use smaller values of $\kappa$, because the central part of instanton-induced potential has almost a linearly rising behavior and then is saturated as the interdistance of the quarks increases [34,35]. It means that the instanton effects reduce the strength of the linear scalar potential. In other words, the instanton-induced interactions contribute partially to the confining potential in a general form:

$V_{\text {conf }}(r)=$ const $+\kappa r+$ possible nonlinear terms.

For example, the leading order contribution from instantons in Eq. (8) to the total $Q \bar{Q}$ potential at small distances can be treated as a nonlinear correction to the linearly rising potential. In this context, the instanton-induced potential at large distances $\left(4 \pi \rho^{3} / N_{c} R^{4}\right) \mathcal{I}_{0}$ has a meaning of partial contribution to the constant part of the confining potential in Eq. (38).

Finally, we discuss the smearing parameter $\sigma$ that was introduced to avoid the singular behavior of the pointlike spin-spin interactions. Its value gets smaller if the instanton-induced potentials are included. Note that the pointlike interaction is actually an artifact of an $\mathcal{O}\left(v_{q}^{2} / c^{2}\right)$ expansion of the $T$-matrix [54]. Introducing the instantoninduced potential, ${ }^{1}$ one can partially solve the divergence problem that arises from the singular spin-spin interactions.

\footnotetext{
${ }^{1}$ Note that the spin-dependent parts of the instanton-induced potentials are regular.
} 


\section{SUMMARY AND OUTLOOK}

In the present work, we aimed at investigating the instanton effects on the charmonium mass spectrum, based on a nonrelativistic potential model. Though we fixed the relevant parameters by using the masses of the six $S$-wave charmonia, we did not intend to carry out the fine-tuning of the parameters. The results showed that the instantoninduced potentials improved the mass spectrum with the original values of the average size of the instanton and the average interdistance between instantons. We discussed also the physical implications of the parameters such as the charm-quark mass, the running strong coupling constant, the string tension, and the smearing factor. The instantoninduced potentials enable one to understand more clearly these physical parameters.

Though we considered certain nonperturbative contributions to the mass spectrum of the charmonia from the instanton vacuum, we still need to take into account yet additional effects from the instantons. Recently, it was shown that the instanton effects or the screening effects in the Coulomb-like potential of one-gluon exchange will appear due to gluon propagation in instanton media [55]. Since the gluon is screened by the instanton effects and as a result it acquires an effective mass, we have in addition a
Yukawa-type potential between the heavy quark and heavy antiquark. One needs to examine how this screened potential may influence the charmonium masses.

Another interesting issue is related to strong decays of excited charmonia, which involve the pions. Since the pion is the pseudo-Goldstone boson that appears from the spontaneous breakdown of chiral symmetry, the instanton effects will come into critical play in describing these decay processes. Related works are under way.

\section{ACKNOWLEDGMENTS}

We are grateful to P. Gubler, A. Hosaka, T. Maruyama, M. Oka, and M. Musakhanov for useful discussions. H.-Ch. K. wants to express his gratitude to the members of the Advanced Science Research Center (ASRC) at the Japan Atomic Energy Agency (JAEA) for the hospitality, where part of the present work was done. This work is supported by the Basic Science Research Program through the National Research Foundation (NRF) of Korea funded by the Korean government (Ministry of Education, Science and Technology, MEST), Grants No. 2016R1D1A1B03935053 (U. Y.), No. 2018R1A2B2001752 (H.-Ch. K.), and No. 2018R1A5A1025563 (H.-Ch. K.). The work was also partly supported by the RIKEN iTHES Project.
[1] S. K. Choi et al. (Belle Collaboration), Phys. Rev. Lett. 91, 262001 (2003).

[2] B. Aubert et al. (BABAR Collaboration), Phys. Rev. D 71, 071103 (2005).

[3] B. Aubert et al. (BABAR Collaboration), Phys. Rev. Lett. 95, 142001 (2005).

[4] K. Abe et al. (Belle Collaboration), Phys. Rev. Lett. 98, 082001 (2007).

[5] S. K. Choi et al. (Belle Collaboration), Phys. Rev. Lett. 100, 142001 (2008).

[6] A. Bondar et al. (Belle Collaboration), Phys. Rev. Lett. 108, 122001 (2012).

[7] Z. Q. Liu et al. (Belle Collaboration), Phys. Rev. Lett. 110, 252002 (2013).

[8] M. Ablikim et al. (BESIII Collaboration), Phys. Rev. Lett. 110, 252001 (2013).

[9] M. Ablikim et al. (BESIII Collaboration), Phys. Rev. Lett. 111, 242001 (2013).

[10] R. Aaij et al. (LHCb Collaboration), Phys. Rev. Lett. 110, 222001 (2013).

[11] M. Ablikim et al. (BESIII Collaboration), Phys. Rev. Lett. 112, 022001 (2014).

[12] R. Aaij et al. (LHCb Collaboration), Phys. Rev. Lett. 112, 222002 (2014).

[13] R. Aaij et al. (LHCb Collaboration), Phys. Rev. D 92 , 112009 (2015).
[14] C. Z. Yuan (BESIII Collaboration), Front. Phys. 10, 101401 (2015).

[15] C. Z. Yuan (Belle Collaboration), AIP Conf. Proc. 1735, 020010 (2016).

[16] E. S. Swanson, Phys. Rep. 429, 243 (2006).

[17] E. Eichten, S. Godfrey, H. Mahlke, and J. L. Rosner, Rev. Mod. Phys. 80, 1161 (2008).

[18] M. B. Voloshin, Prog. Part. Nucl. Phys. 61, 455 (2008).

[19] N. Brambilla et al., Eur. Phys. J. C 71, 1534 (2011).

[20] S. L. Olsen, Front. Phys. 10, 101401 (2015).

[21] H. X. Chen, W. Chen, X. Liu, Y. R. Liu, and S. L. Zhu, Rep. Prog. Phys. 80, 076201 (2017).

[22] E. Eichten, K. Gottfried, T. Kinoshita, J. B. Kogut, K. D. Lane, and T. M. Yan, Phys. Rev. Lett. 34, 369 (1975); 36, 1276 (1976).

[23] E. Eichten, K. Gottfried, T. Kinoshita, K. D. Lane, and T. M. Yan, Phys. Rev. D 17, 3090 (1978); 21, 313 (1980).

[24] L. Susskind, in Proceedings of the Weak and Electromagnetic Interactions at High Energies, edited by R. Balian and C. H. Llewellyn Smith (North-Holland, New York, 1977).

[25] T. Appelquist, M. Dine, and I. J. Muzinich, Phys. Lett. 69B, 231 (1977).

[26] T. Appelquist, M. Dine, and I. J. Muzinich, Phys. Rev. D 17, 2074 (1978).

[27] W. Fischler, Nucl. Phys. B129, 157 (1977).

[28] M. Peter, Phys. Rev. Lett. 78, 602 (1997). 
[29] M. Peter, Nucl. Phys. B501, 471 (1997).

[30] Y. Schroder, Phys. Lett. B 447, 321 (1999).

[31] A. V. Smirnov, V. A. Smirnov, and M. Steinhauser, Phys. Rev. Lett. 104, 112002 (2010).

[32] C. Anzai, Y. Kiyo, and Y. Sumino, Phys. Rev. Lett. 104, 112003 (2010).

[33] K. G. Wilson, Phys. Rev. D 10, 2445 (1974).

[34] U. T. Yakhshiev, H.-Ch. Kim, M. M. Musakhanov, E. Hiyama, and B. Turimov, Chin. Phys. C 41, 083102 (2017).

[35] D. Diakonov, V. Y. Petrov, and P. V. Pobylitsa, Phys. Lett. B 226, 372 (1989).

[36] D. Diakonov and V. Y. Petrov, Nucl. Phys. B245, 259 (1984).

[37] D. Diakonov and V. Y. Petrov, Nucl. Phys. B272, 457 (1986).

[38] D. Diakonov, Prog. Part. Nucl. Phys. 51, 173 (2003).

[39] E. Eichten and F. Feinberg, Phys. Rev. D 23, 2724 (1981).

[40] E. V. Shuryak, Nucl. Phys. B203, 93 (1982).

[41] H.-Ch. Kim, M. M. Musakhanov, and M. Siddikov, Phys. Lett. B 633, 701 (2006).

[42] K. Goeke, M. M. Musakhanov, and M. Siddikov, Phys. Rev. D 76, 076007 (2007).
[43] K. Goeke, H.-Ch. Kim, M. M. Musakhanov, and M. Siddikov, Phys. Rev. D 76, 116007 (2007).

[44] T. Barnes, S. Godfrey, and E. S. Swanson, Phys. Rev. D 72, 054026 (2005).

[45] M. C. Chu, J. M. Grandy, S. Huang, and J. W. Negele, Phys. Rev. D 49, 6039 (1994).

[46] J. W. Negele, Nucl. Phys. B, Proc. Suppl. 73, 92 (1999).

[47] T. A. DeGrand, Phys. Rev. D 64, 094508 (2001).

[48] P. Faccioli and T. A. DeGrand, Phys. Rev. Lett. 91, 182001 (2003).

[49] C. Quigg and J. L. Rosner, Phys. Rep. 56, 167 (1979).

[50] E. Hiyama, Y. Kino, and M. Kamimura, Prog. Part. Nucl. Phys. 51, 223 (2003).

[51] S. Chernyshev, M. A. Nowak, and I. Zahed, Phys. Lett. B 350, 238 (1995).

[52] M. Tanabashi et al. (Particle Data Group), Phys. Rev. D 98, 030001 (2018).

[53] G. S. Bali, Phys. Rep. 343, 1 (2001).

[54] T. Barnes and G. I. Ghandour, Phys. Lett. 118B, 411 (1982).

[55] M. Musakhanov and O. Egamberdiev, Phys. Lett. B 779, 206 (2018). 